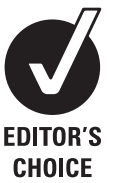

CHOICE
${ }^{1}$ Cardiology Department, Maria Vittoria Hospital, Torino, Italy ${ }^{2}$ Ospedali Riuniti, Bergamo, Italy ${ }^{3}$ Cardiac Surgery, Ospedale Mauriziano, Torino, Italy ${ }^{4}$ Sheba Medical Center, Tel Hashomer and Sackler Faculty of Medicine, Tel Aviv University, Israel

\section{Correspondence to}

Massimo Imazio, Cardiology Department, Maria Vittoria Hospital, via Cibrario 72, 10141 Torino, Italy; massimo_imazio@yahoo.it

Received 30 October 2011 Revised 12 January 2012 Accepted 7 February 2012 Published Online First 22 March 2012

\title{
Efficacy and safety of colchicine for pericarditis prevention. Systematic review and meta-analysis
}

\author{
Massimo Imazio, ${ }^{1}$ Antonio Brucato, ${ }^{2,3}$ Davide Forno, ${ }^{1}$ Silvia Ferro, ${ }^{1}$ Riccardo Belli, ${ }^{1}$ \\ Rita Trinchero, ${ }^{1}$ Yehuda Adler ${ }^{4}$
}

\section{ABSTRACT}

Objectives The purpose of this study was to investigate the efficacy and safety of colchicine for pericarditis prevention.

Background Recurrent pericarditis prevention is a major management goal that may reduce morbidity and management costs. Although empiric anti-inflammatory therapy is considered the mainstay of treatment, no specific drug has been proven to be efficacious for prevention but colchicine.

Methods Controlled clinical studies were searched in several databases and were included provided they focused on the pharmacologic primary or secondary prevention of pericarditis. We performed a meta-analysis including studies primary outcome, adverse events, and drug withdrawal.

Results From the initial sample of 127 citations, five controlled clinical trials were finally included $(795$ patients): three studies were double-blind randomised controlled trials, and two studies were open-label randomised controlled trials. Trials followed patients for a mean of 13 months. Meta-analytic pooling showed that colchicine use was associated with a reduced risk of pericarditis during follow-up $(\mathrm{RR}=0.40,95 \% \mathrm{Cl} 0.30$ to 0.54 , p for effect $<0.001$, p for heterogeneity $=0.95$, $\mathrm{I}^{2}=0 \%$ ) either for primary or secondary prevention without a significant higher risk of adverse events compared with placebo $(\mathrm{RR}=1.22,95 \% \mathrm{Cl} 0.71$ to 2.10, p for effect $0.48, p$ for heterogeneity $=0.44$, $\left.\mathrm{I}^{2}=0 \%\right)$, but more cases of drug withdrawals $(\mathrm{RR}=1.85$, $95 \% \mathrm{Cl} 1.04$ to $3.29, \mathrm{p}$ for effect $0.04, \mathrm{p}$ for heterogeneity $=0.42, \mathrm{I}^{2}=0 \%$ ). Gastrointestinal intolerance is the most frequent side effect (mean incidence $8 \%$ ), but no severe adverse events were recorded.

Conclusions Available evidence suggests that colchicine is safe and efficacious for the primary and secondary prevention of pericarditis.

Optimal medical management of pericarditis is a relatively common clinical problem; especially prevention of recurrences is a difficult issue. Recurrent pericarditis has been reported in $25 \%$ to $50 \%$ of cases, ${ }^{12}$ often the most troublesome and common complication of pericarditis. ${ }^{3}$ Recurrences are often a cause of re-hospitalisation and repetition of diagnostic tests. On this basis, prevention of pericarditis is a treatment goal that may improve the quality of life of patients and reduce disease management costs.

Unfortunately, the pathogenesis of recurrences is not well known, although recent published evidence of serum anti-heart (AHA) and anti-intercalated-disk (AIDA) in patients with idiopathic recurrent pericarditis, strengthens the autoimmune etiopathogenesis of these forms, often suspected to have an immune-mediated origin. ${ }^{4}$ Thus, in clinical practice, using a conventional diagnostic approach, most cases of acute or recurrent pericarditis are labelled as 'idiopathic'. Idiopathic, viral, postoperative pericarditis in the setting of the postpericardiotomy syndrome after cardiac surgery, as well as uncomplicated pericarditis related to a systemic inflammatory disease $e^{5}$ have similar empiric anti-inflammatory therapies, that are considered the mainstay of treatment for pericarditis. ${ }^{3}$ No specific drug has been proven to be efficacious for prevention beyond treatment, with the possible important exception of colchicine. ${ }^{6} 7$

On this basis, we aimed to summarise the available data on pharmacologic primary and secondary prevention of pericarditis by colchicine and determine its efficacy and safety in available clinical trials. To the best of our knowledge this is the first systematic review on this issue.

\section{METHODS}

\section{Selection criteria and search strategy}

We included any randomised clinical trial on pharmacological prevention of pericarditis. Potentially relevant studies published up to December 2011 were searched in BioMedCentral, the Cochrane Collaboration Database of Randomised Trials (CENTRAL), ClinicalTrials.gov, EMBASE, Google Scholar, MEDLINE/PubMed, and Scopus. The PubMed search was performed with the term 'pericarditis' and 'colchicine'. Recent (2005 or later) conference proceedings from the American Heart Association, American College of Cardiology, and the European Society of Cardiology were electronically or manually searched. Searches were not limited by language, sex, or age. In addition, references of retrieved studies were scanned for additional unpublished studies.

\section{Study selection and data collection}

Initially retrieved references were checked at the title/abstract level for pertinence. Potentially pertinent studies were retrieved as full reports for further appraisal according to the following selection criteria. Inclusion criteria to be fulfilled for data extraction were: (1) controlled clinical trial, (2) head-to head comparisons of treatments or versus placebo, (3) for the primary or secondary prevention of pericarditis. Final study selection for inclusion in 
the review was performed by two independent reviewers (DF and SF), with divergences resolved by consensus.

Study evaluation included general methodological quality features, including sequence-generation, blinding, use of intention-to-treat analysis, completeness of follow-up, and allocation concealment. ${ }^{8} 9$

Baseline, outcome, and adverse events data were retrieved from included studies, focusing on the pharmacologic treatment (dose, timing, and duration of administration), primary and secondary clinical end points, and adverse events. We extracted data on the incidence of pericarditis and recurrences. We also extracted data on adverse events including gastrointestinal intolerance, increases in AST and ALT, CK, myotoxicity, alopecia, or any possible side effect related to colchicine. Data on drug withdrawal were recorded and included in the meta-analysis.

Data abstraction and validity/risk of bias appraisal were performed on pre-specified electronic forms by two independent reviewers (DF and SF), with divergences resolved by consensus.

\section{Data analysis}

In order to assess inter-rater reliability on inclusion of articles, we calculated the $\phi$ statistic, which provides a measure of interobserver agreement independent of chance.

Categorical variables are reported as percentages. Statistical homogeneity and consistency were checked by means of $\chi^{2}$ test and $\mathrm{I}^{2} . \mathrm{I}^{2}$ is computed as $100 \% \times(\mathrm{Q}-\mathrm{df}) / \mathrm{Q}$, where $\mathrm{Q}$ is Cochran heterogeneity statistic and $\mathrm{df}$ the degrees of freedom. A value of $0 \%$ indicates no observed heterogeneity, and larger values show increasing heterogeneity. We calculated the RR and appropriate $95 \%$ CI of outcomes, adverse events, and drug withdrawal according to the number of events reported in the original studies on an intention-to-treat basis. Computations were carried out with RevMan V.5.0 (The Nordic Cochrane Centre, Copenhagen, Denmark) and the software package SPSS V.13.0.

\section{RESULTS}

Figure 1 displays the flow diagram of study inclusion. From initial sample of 127 citations, 14 citations were appraised in complete form for more detailed evaluation, leading to the final inclusion of five controlled clinical trials (treatment comparison was colchicine vs placebo) for the prevention of pericarditis (table 1). ${ }^{1} \quad 2 \quad 10-12$ Included studies enrolled a total of 795 patients. The inter-rater reliability for study inclusion was very good $(\phi>0.9)$.

Baseline features of the selected studies are reported in table 2: two studies were aimed at the primary prevention of postoperative pericarditis, ${ }^{10} 11$ and the other three for the secondary prevention of pericarditis. ${ }^{12} 12$ Study quality was relatively high with a low overall risk of bias (figure 2). All studies reported on who was blinded in the trial, and provided follow-up data, adequate methods of randomisation sequence generation, concealed allocation of study participants, and intention-to-treat analysis in 4 of 5 (80\%). ${ }^{12} 1112$

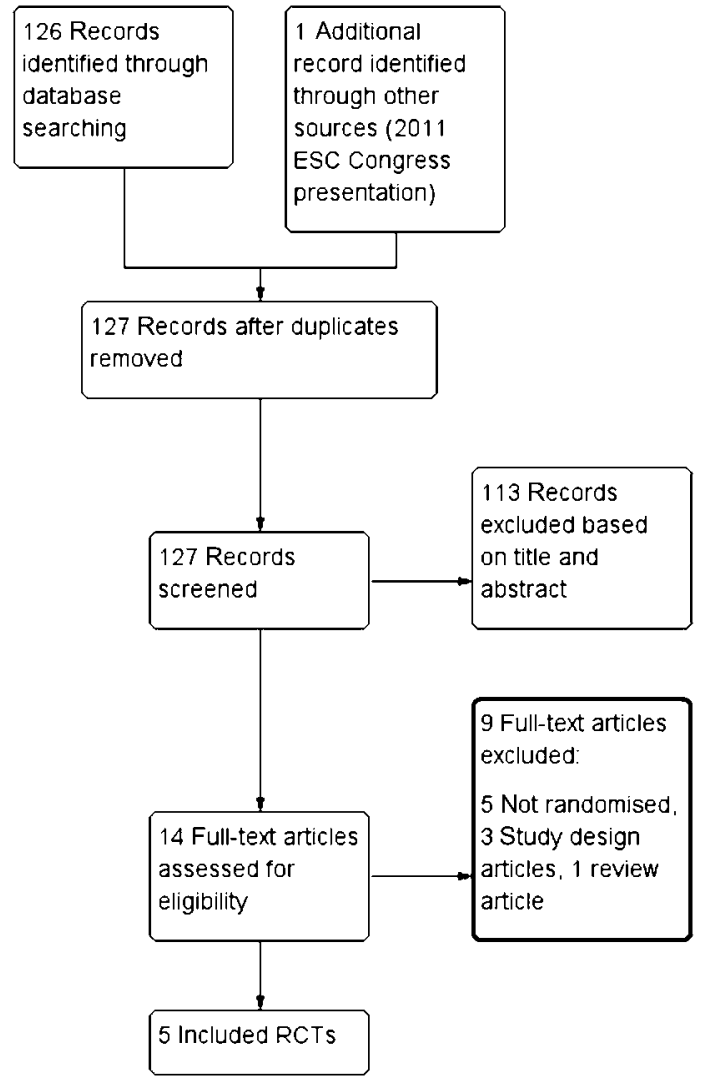

Figure 1 Flow diagram of included studies.

\section{Pericarditis prevention}

Trials followed patients for a mean of 13 months. Meta-analytic pooling showed that colchicine use was associated with a reduced risk of pericarditis during follow-up $(R R=0.40,95 \% \mathrm{CI}$ 0.30 to 0.54 , p for effect $<0.001, \mathrm{p}$ for heterogeneity $=0.95$, $\mathrm{I}^{2}=0 \%$ ) either for primary ( $\mathrm{R} R=0.44,95 \%$ CI 0.27 to 0.70 , p for effect $<0.001$, p for heterogeneity $=0.80, \mathrm{I}^{2}=0 \%$ ) or secondary prevention $(\mathrm{RR}=0.39,95 \% \mathrm{CI} 0.27$ to 0.56 , p for effect $<0.001, \mathrm{p}$ for heterogeneity $=0.77, \mathrm{I}^{2}=0 \%$ ). Colchicine was efficacious either for the primary or secondary prevention of pericarditis (figure 3). Inspection of the funnel plot did not suggest the presence of publication bias.

\section{Adverse events and drug withdrawal}

One clinical trial was excluded from analysis because 52 of 163 patients $(31.9 \%)$ were excluded from subsequent outcome assessment for side effects or other complications. ${ }^{10}$ Metaanalytic pooling showed that colchicine use was not associated with an increased risk of side effects compared with placebo $(\mathrm{RR}=1.22,95 \%$ CI 0.71 to 2.10 , p for effect 0.48 , p for heterogeneity $=0.44, \mathrm{I}^{2}=0 \%$ ) (figure 4 ). The most common reported side effect was gastrointestinal intolerance that was associated

Table 1 Mean features of included studies

\begin{tabular}{|c|c|c|c|c|c|c|}
\hline Study & Location/year & Design & Setting & Therapeutic class & Patients Number* & Follow-up (months) \\
\hline Finkelstein $\mathrm{Y}$ et $a l^{10}$ & Israel/2002 & Double-blind RCT & Multicenter & Colchicine & 111 & 3 \\
\hline COPE study ${ }^{1}$ & Italy/2005 & Open-label RCT & Single center & Colchicine & 120 & 18 \\
\hline CORE study ${ }^{2}$ & Italy/2005 & Open-label RCT & Single center & Colchicine & 84 & 18 \\
\hline COPPS study ${ }^{11}$ & Italy/2010 & Double-blind RCT & Multicenter & Colchicine & 360 & 12 \\
\hline CORP study ${ }^{12}$ & Italy/2011 & Double-blind RCT & Multicenter & Colchicine & 120 & 18 \\
\hline
\end{tabular}

*Real study population included for results analysis in the original study.

$\mathrm{RCT}$, randomised clinical trial. 
Table 2 Baseline features of patients in included studies

\begin{tabular}{|c|c|c|c|}
\hline Study & $\begin{array}{l}\text { Mean } \\
\text { age (years) }\end{array}$ & Male (\%) & Intervention \\
\hline $\begin{array}{l}\text { Finkelstein Y } \\
\text { et } a l^{10}\end{array}$ & 64 & 81/111 (73.0) & $\begin{array}{l}\text { Colchicine } 1.5 \mathrm{mg} / \mathrm{day} \\
\text { (in three doses) from } 3 \mathrm{rd} \\
\text { postoperative day for } 1 \text { month }\end{array}$ \\
\hline COPE study ${ }^{1}$ & 57 & $54 / 120(45.0)$ & $\begin{array}{l}\text { Colchicine } 1.0-2.0 \mathrm{mg} \text { for } \\
\text { the first day followed by a } \\
\text { maintenance dose of } \\
0.5-1.0 \mathrm{mg} \text { daily for } 3 \text { months* }\end{array}$ \\
\hline CORE study ${ }^{2}$ & 51 & $29 / 84(34.5)$ & $\begin{array}{l}\text { Colchicine } 1.0-2.0 \mathrm{mg} \text { for } \\
\text { the first day followed by a } \\
\text { maintenance dose of } \\
0.5-1.0 \mathrm{mg} \text { daily for } 6 \text { months }\end{array}$ \\
\hline COPPS study ${ }^{11}$ & 66 & $239 / 360(66.4)$ & $\begin{array}{l}\text { Colchicine } 1.0-2.0 \mathrm{mg} \text { for the } \\
\text { first day followed by a } \\
\text { maintenance dose of } \\
0.5-1.0 \mathrm{mg} \text { daily for } 1 \text { month* }\end{array}$ \\
\hline CORP study ${ }^{12}$ & 48 & $63 / 120(52.5)$ & $\begin{array}{l}\text { Colchicine } 1.0-2.0 \mathrm{mg} \text { for } \\
\text { the first day followed by a } \\
\text { maintenance dose of } \\
0.5-1.0 \mathrm{mg} \text { daily for } 6 \text { months* }\end{array}$ \\
\hline
\end{tabular}

*Highest dose in patients $\geq 70 \mathrm{~kg}$, and the lowest dose for patients $<70 \mathrm{~kg}$ or intolerant to the highest dose. Colchicine was given as adjunct to aspirin or NSAID in acute and recurrent pericarditis (COPE, CORE, and CORP studies).

with drug withdrawal in almost all cases. Colchicine use is associated with an increased risk of drug withdrawal $(R R=1.85$, $95 \%$ CI 1.04 to 3.29 , p for effect 0.04 , p for heterogeneity $=0.42$, $\mathrm{I}^{2}=0 \%$ ) (figure 5). A severe adverse event was considered an untoward event which was fatal or life-threatening or required hospitalisation or was significantly or permanently disabling or medically significant (may jeopardise the patient and may require medical or surgical intervention to prevent an adverse outcome). No severe side effects were reported in clinical trials. A detailed list of all reported side effects together with colchicine doses and study exclusion criteria is reported in table 3 .

\section{DISCUSSION}

Recurrent pericarditis is the most troublesome complication of pericarditis. Although different anti-inflammatory agents are used for treatment (ie, acetylsalicylic acid, indomethacin, steroids, and colchicine), only colchicine has been proven to be efficacious for prevention. In the present systematic review, multiple databases were searched without language restrictions leading to the selection of five controlled clinical studies on colchicine use for the prevention of pericarditis. Colchicine was safe and efficacious in all randomised clinical trials on adult

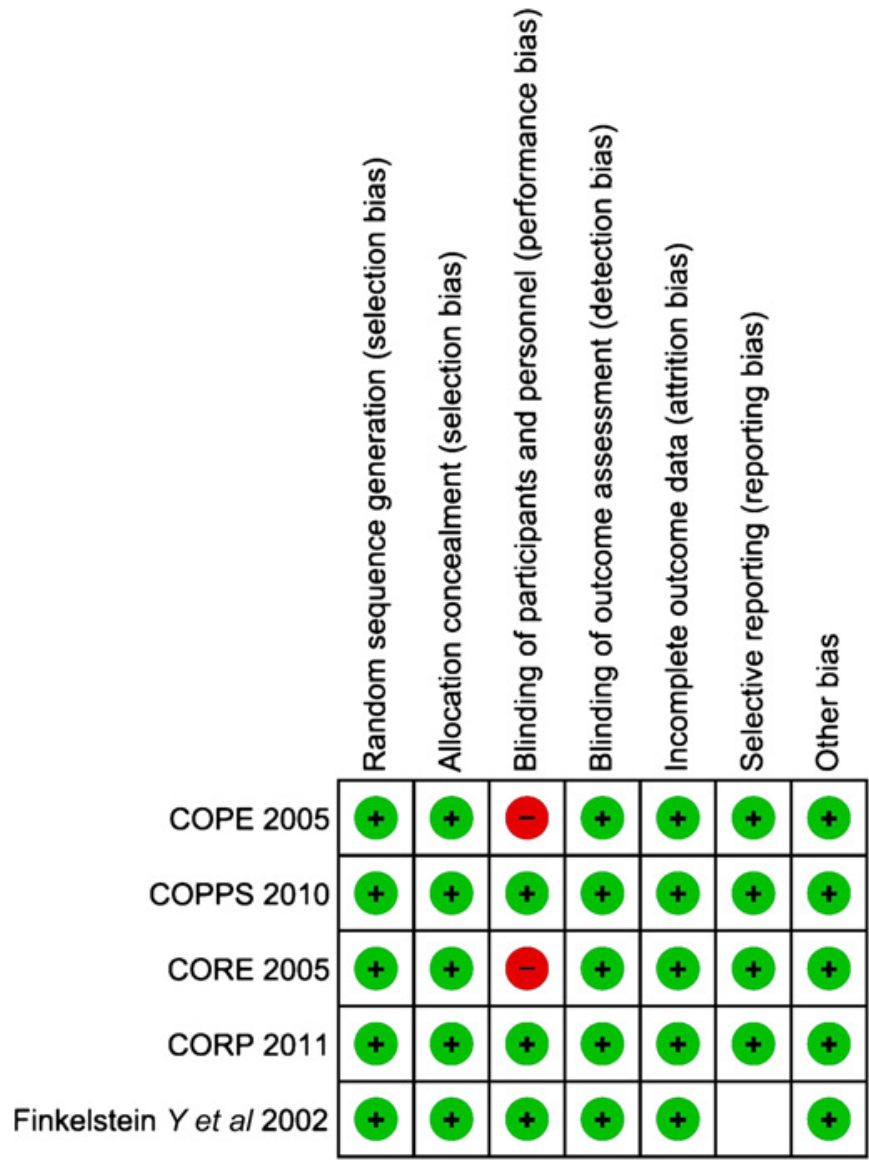

Figure 2 Risk of bias summary: review authors' judgements about each risk of bias item for each included study. Empty boxes indicate unclear risk of bias. The green circle indicates a low risk of bias, the red circle identifies potential risk of bias (in the figure specifically related to the open-label randomised trial design of the COPE and CORE studies).

patients for the primary and secondary prevention of pericarditis $(\mathrm{RR}=0.40,95 \% \mathrm{CI} 0.30$ to $0.54 ; \mathrm{p}<0.001)$ without a significant increase of side effects compared to placebo $(R R=1.22,95 \% \mathrm{CI}$ 0.71 to $2.10, \mathrm{p}=0.48$ ), but a higher risk of drug withdrawal $(\mathrm{RR}=1.85,95 \%$ CI 1.04 to 3.29$), \mathrm{p}=0.04)$. Colchicine concentrates in white blood cells, particularly neutrophils, inhibiting tubulin polymerisation, thus interfering with migration and
Figure 3 Forest plot for the risk of pericarditis. M-H, Mantel-Haenszel.

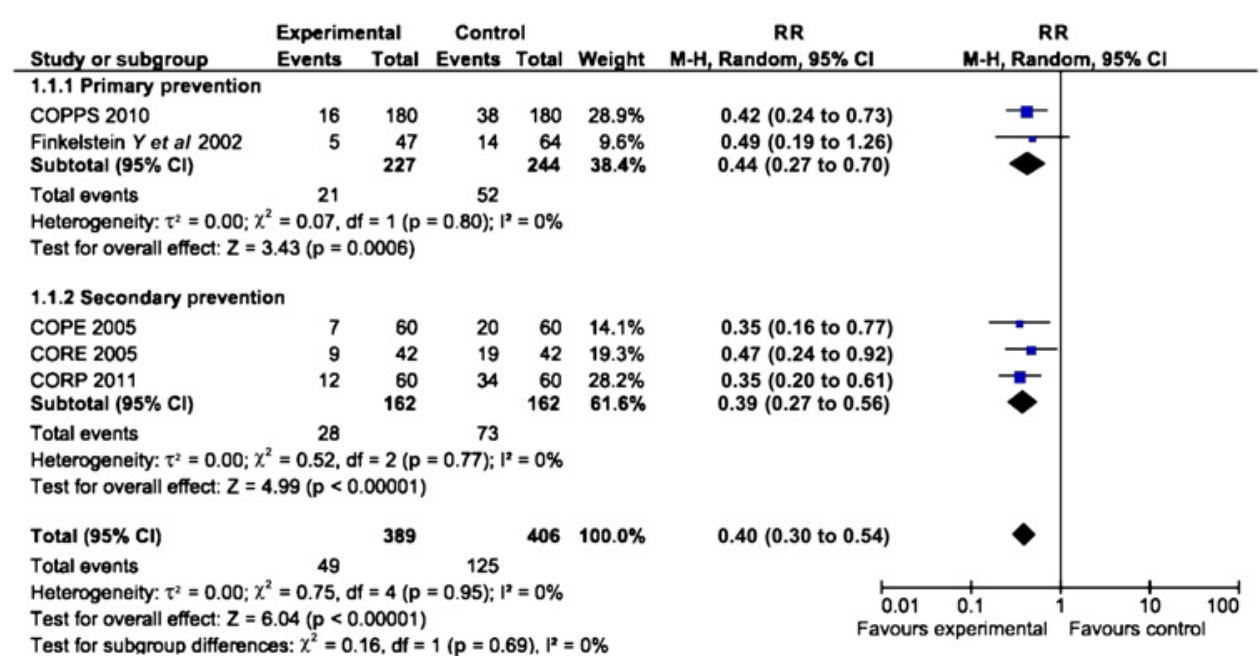


Figure 4 Forest plot for the risk of adverse events.

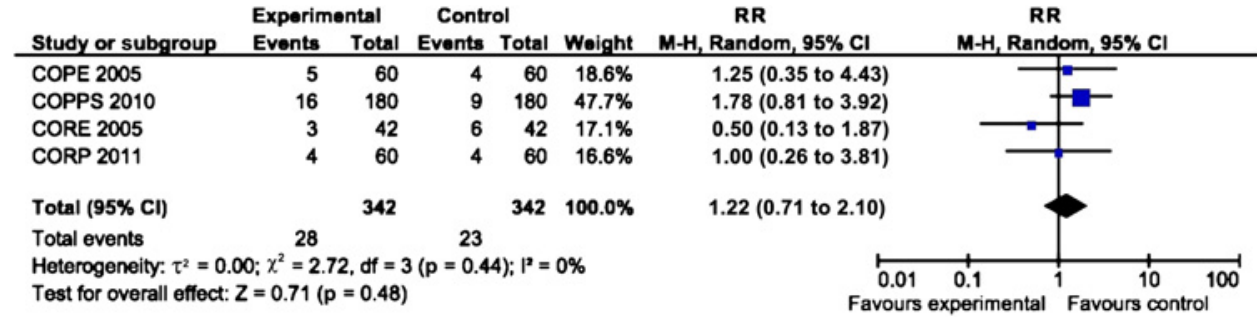

Random, $95 \% \mathrm{Cl}$

Favours experimental Favours control phagocytosis, and reducing the inflammatory cycle. ${ }^{3} \quad 6 \quad 7$ Although the exact number of responders is unknown, the drug has been successfully used for the treatment and prevention of recurrences and to taper corticosteroids in patients with combined therapies including steroids with non-steroidal anti-inflammatory drugs. ${ }^{7}$ However, a careful monitoring of possible contraindications, drug interactions and side effects is necessary. ${ }^{5} 1314$ Colchicine shows a large interindividual bioavailability. Furthermore, interactions with drugs interfering with CYP3A4 dependent enzymes and P-glycoprotein occur and are clinically important. The drug has a narrow therapeutictoxicity window, and potentially serious drug-drug interactions (eg, with clarithromycin and cyclosporine) are better recognised and therefore preventable. The dosage of colchicine must be reduced in patients with relevant hepatic and/or renal dysfunction, and the elderly. However, when appropriately used and contraindications have been excluded, oral colchicine at 0.5-1.0 mg has been shown to be a safe treatment. ${ }^{1} 2$ 10-12

However safe use of the drug requires careful consideration of potential contraindications. Colchicine is indicated for patients without bacterial or neoplastic pericarditis and patients were excluded from clinical trials in case of known severe liver disease or current transaminases $>1.5$ times the upper normal limit, current serum creatinine above $2.5 \mathrm{mg} / \mathrm{dl}$, known myopathy or current serum creatine kinase above the upper normal limit, known blood dyscrasias or gastrointestinal disease, pregnant and lactating women or women of childbearing potential not protected by a contraception method, known hypersensitivity to colchicine. ${ }^{1} 21112$

Use of weight adjusted doses $(0.5 \mathrm{mg}$ as maximum dose for patients $<70 \mathrm{~kg}$ ) and avoidance of attack doses $\geq 1.0 \mathrm{mg}$ daily may be useful to reduce gastrointestinal intolerance. ${ }^{5} \mathrm{~A}$ higher rate of gastrointestinal side effects has been reported with daily doses $>1.0 \mathrm{mg}$ in the study by Finkelstein Y et al (table 3).

Previous reviews on the topic have suggested the safety and efficacy of the drug for pericarditis treatment based on expert opinion and previous retrospective or non-randomised observations. A previous meta-analysis on medical treatments for pericarditis has demonstrated that colchicine use was associated with a reduced risk of treatment failure $(\mathrm{OR}=0.23,95 \%$ CI 0.11 to 0.49 ) and recurrent pericarditis ( $\mathrm{OR}=0.39,95 \%$ CI 0.20 to
$0.77)$, but with a trend towards more adverse effects $(\mathrm{OR}=5.27$, 95\% CI 0.86 to 32.16$).{ }^{15}$ However this meta-analysis did not include recently published clinical trials. ${ }^{11} 12$ In another metaanalysis focused on the prevention of the post-pericardiotomy syndrome, colchicine use was associated a decreased risk of the syndrome $(\mathrm{OR}=0.38,95 \% \mathrm{CI} 0.22$ to 0.65$) .{ }^{16}$ However this meta-analysis did not include clinical trials for secondary prevention of pericarditis not related to the post-pericardiotomy syndrome.

The present meta-analysis focused on prevention of pericarditis by colchicine, includes all published clinical trials and extends current knowledge showing either efficacy or safety of the drug when used at low doses and with appropriate indications and contraindications. However colchicine use is associated with more cases of drug withdrawal because of gastrointestinal intolerance. On this basis, the present metaanalysis provides a stronger evidence base for the use of colchicine in patients with pericarditis, as outlined in previous recommendations on colchicine use in the 2004 guidelines on the management of pericardial diseases of the European Society of Cardiology, based on expert consensus while randomised trials were not available at that time. ${ }^{17}$ At present, there are no available updates of 2004 guidelines, and no specific guidelines on the management of pericardial diseases have been issued by the American College of Cardiology, and the American Heart Association. This meta-analysis is useful to summarise data from all published clinical trials on pericarditis prevention by colchicine.

In conclusion, our study found that colchicine $0.5-1.0 \mathrm{mg}$ daily was safe and efficacious for the primary and secondary prevention of pericarditis and should be considered as first line therapy for pericarditis prevention.

\section{Study strengths}

At present, this is the first comprehensive meta-analysis on this topic, including all published clinical trial up to December 2011. We believe that a rigorous search strategy has resulted in a comprehensive systematic review and meta-analysis on the efficacy and safety of colchicine for pericarditis prevention. Study quality was relatively high with a low overall risk of bias and the level of heterogeneity was negligible.
Figure 5 Forest plot for the risk of drug withdrawal.

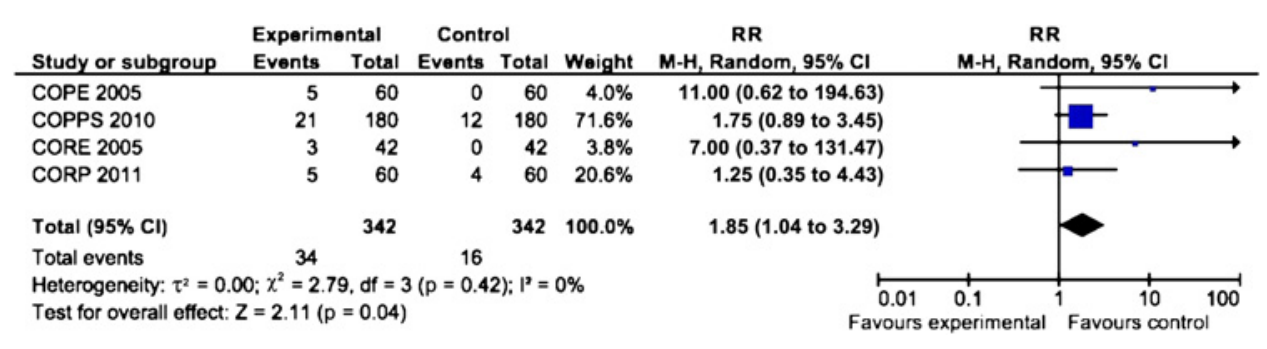


Table 3 Adverse events reported in clinical trials

\begin{tabular}{llllll}
\hline & $\begin{array}{l}\text { Finkelstein } \\
\text { et } \mathbf{a l}^{\mathbf{1 0}}\end{array}$ & $\begin{array}{l}\text { COPE } \\
\text { study }\end{array}$ & $\begin{array}{l}\text { CORE } \\
\text { study }^{2}\end{array}$ & $\begin{array}{l}\text { COPPS } \\
\text { study }^{11}\end{array}$ & $\begin{array}{l}\text { CORP } \\
\text { study }^{12}\end{array}$ \\
\hline $\begin{array}{l}\text { Gastrointestinal } \\
\text { Alopecia }\end{array}$ & NA $^{*}$ & 6.7 vs 8.3 & 14.0 vs 7.0 & 4.4 vs 8.9 & 5.0 vs 7.0 \\
NA & 0 vs 0 & 0 vs 0 & 0 vs 0 & 0 vs 0 \\
Hepatoxia & NA & 0 vs 0 & 0 vs 0 & 0 vs 0 & 0 vs 0 \\
$\begin{array}{l}\text { Myotoxicity } \\
\text { Bone marrow }\end{array}$ & NA & 0 vs 0 & 0 vs 0 & 0 vs 0 & 2.0 vs 0 \\
toxicity & NA & 0 vs 0 & 0 vs 0 & 1 vs 0 & 0 vs 0 \\
Drug withdrawal & NA & 0 vs 0 & 0 vs 0 & 0 vs 0 & 0 vs 0 \\
\hline
\end{tabular}

Data are reported as percentages with comparison placebo versus colchicine. For all comparison $p$ values $>0.05$.

*52 of $163(31.9 \%)$ were excluded from analysis of outcomes for side effects or other complications.

NA, not available.

\section{Study limitations}

Some of the included trials were open label, which might have introduced bias; however our findings were similar in open-label and placebo-controlled trials. However all trials have independent blinded outcome assessment with very low or absent participant dropout, thus indicating studies of high quality. Although an apparently heterogeneous population (idiopathic, viral, postoperative pericarditis as well as pericarditis related to a systemic inflammatory disease) has been included in the present systematic review, the same treatment and preventive strategies are adopted and recommended for such patients, that are heterogeneous for aetiology but homogenous for pericarditis medical therapy. Only bacterial and neoplastic pericarditis has been excluded because requiring specific treatments. The last potential limitation of the present study is the inclusion of trials exploring primary and secondary prevention of pericarditis. However the aim of the meta-analysis was to provide a comprehensive review of efficacy and safety of colchicine for both primary and secondary prevention, thus comparing and summarising both the effects. We think that this updated review may be useful in the clinical setting and provides a stronger evidence base for future recommendations and clinical research in the field.

\section{CONCLUSIONS}

At present colchicine is the only drug that has been proven efficacious and safe for pericarditis prevention in clinical trials, with a similar effect for primary and secondary prevention. On this basis, the drug should be considered as a first line agent for pericarditis prevention.

Contributors The authors are solely responsible for the design and conduct of the study, the analysis, the drafting and editing of the paper, and its final contents. All authors contributed the conception, analysis and drafting of the manuscript as well as its critical revision. Moreover all authors gave final approval of the submitted manuscript. The paper is not under consideration elsewhere and none of the paper's contents have been previously published.

Competing interests None.

Provenance and peer review Not commissioned; externally peer reviewed.

\section{REFERENCES}

1. Imazio M, Bobbio $M$, Cecchi $E$, et al. Colchicine in addition to conventional therapy for acute pericarditis: results of the COlchicine for acute PEricarditis (COPE) trial. Circulation 2005;112:2012-16

2. Imazio M, Bobbio M, Cecchi E, et al. Colchicine as first-choice therapy for recurrent pericarditis: results of the CORE (COlchicine for REcurrent pericarditis) trial. Arch Intern Med 2005;165:1987-91.

3. Imazio M, Spodick DH, Brucato A, et al. Controversial issues in the management of pericardial diseases. Circulation 2010:121:916-28.

4. Caforio AL, Brucato A, Doria A, et al. Anti-heart and anti-intercalated disk autoantibodies: evidence for autoimmunity in idiopathic recurrent acute pericarditis. Heart 2010;96:779-84.

5. Imazio M. Pericardial involvement in systemic inflammatory diseases. Heart 2011:97:1882-92.

6. Adler Y, Finkelstein Y, Guindo J, et al. Colchicine treatment for recurrent pericarditis: a decade of experience. Circulation 1998:97:2183-5.

7. Imazio M, Brucato A, Trinchero R, et al. Colchicine for pericarditis: hype or hope? Eur Heart J 2009;30:532-9.

8. Schulz KF, Chalmers I, Hayes RJ, et al. Empirical evidence of bias. Dimensions of methodological quality associated with estimates of treatment effects in controlled trials. JAMA 1995;273:408-12.

9. Higgins JPT, Thompson SG, Deeks JJ, et al. Measuring inconsistency in metaanalyses. BMJ 2003;327:557-60.

10. Finkelstein $\mathbf{Y}$, Shemesh J, Mahlab $\mathrm{K}$, et al. Colchicine for the prevention of postpericardiotomy syndrome. Herz 2002;27:791-4.

11. Imazio M, Trinchero R, Brucato A, et al; COPPS Investigators. COlchicine for the Prevention of the Post-pericardiotomy Syndrome (COPPS): a multicentre, randomized double-blind, placebo-controlled trial. Eur Heart J 2010;31:2749-54.

12. Imazio M, Brucato A, Cemin R, et al; CORP Investigators. COlchicine for recurrent pericarditis (CORP). A randomized, controlled trial. Ann Intern Med 2011;155:409-14.

13. Terkeltaub RA. Colchicine update: 2008. Semin Arthritis Rheum 2009;38:411-19

14. Cocco G, Chu DC, Pandolfi S. Colchicine in clinical medicine. A guide for internists Eur J Intern Med 2010;21:503-8.

15. Lotrionte $\mathbf{M}$, Biondi-Zoccai G, Imazio M, et al. International collaborative systematic review of controlled clinical trials on pharmacologic treatments for acute pericarditis and its recurrences. Am Heart $J$ 2010;160:662-70.

16. Imazio M, Brucato A, Markel G, et al. Meta-analysis of randomized trials focusing on prevention of the postpericardiotomy syndrome. Am J Cardiol 2011:108:575-9.

17. Maisch B, Seferović PM, Ristić AD, et al; Task Force on the Diagnosis and Management of Pericardial Diseases of the European Society of Cardiology. Guidelines on the diagnosis and management of pericardial diseases. Eur Heart $J$ 2004;25:587-610. 